04

\title{
Первые результаты исследований ионного теплопереноса на сферическом токамаке Глобус-М2
}

\author{
(C) А.Ю. Тельнова, И.В. Мирошников, М.М. Митранкова, Н.Н. Бахарев, \\ В.К. Гусев, Н.С. Жильцов, Е.О. Киселев, Г.С. Курскиев, В.Б. Минаев, Ю.В. Петров, \\ Н.В. Сахаров, П.Б. Щёголев, Е.А. Тюхменева
}

Физико-технический институт им. А.Ф. Иофффе РАН, Санкт-Петербург, Россия

E-mail: anna.telnova@mail.ioffe.ru

Поступило в Редакцию 16 ноября 2020г.

В окончательной редакции 9 января 2021г.

Принято к публикации 5 фревраля 2021 г.

Представлены результаты первых измерений профиля ионной температуры на новом сферическом токамаке Глобус-М2, полученные в разрядах с нейтральной инжекцией с помощью диагностики спектроскопии перезарядки и анализатора спектра атомов перезарядки. На основании полученных экспериментальных профилей ионной температуры с помощью кода ASTRA проведено моделирование, результаты которого указывают на преимущественно неоклассический характер ионного теплопереноса.

Ключевые слова: термоядерный синтез, транспортный анализ, нагрев плазмы с помощью нейтральной инжекции, ионный теплоперенос, сферический токамак.

DOI: 10.21883/PJTF.2021.09.50903.18618

Исследование процессов переноса в токамаке представляет собой одну из фундаментальных задач в современной физике плазмы, решение которой позволит приблизиться к практическому использованию термоядерных установок. Преимуществом сферических токамаков (помимо компактных размеров и соответственно меньшей стоимости) является преобладание неоклассического теплопереноса ионов в Н-режиме [1-6] (а для токамака Глобус-М в некоторых случаях и в Lрежиме [7]), т.е. меньших потерь тепла из плазмы, по сравнению с классическими токамаками, где преобладает аномальный перенос ионов. Глобус-М2 - новый компактный сферический токамак [8], который является модернизированной версией токамака Глобус-М [9] (с аспектным отношением $A=1.5$, большим радиусом $R=0.36 \mathrm{~m}$, малым радиусом $a=0.24 \mathrm{~m})$. В результате реконструкции проектные параметры установки были двукратно увеличены, и тороидальное магнитное поле $B_{T}$ и ток плазмы $I_{P}$ могут быть увеличены до $1 \mathrm{~T}$ и $500 \mathrm{kA}$ соответственно. Помимо модернизации электромагнитной системы и систем питания был расширен диагностический комплекс установки [10]. Одними из ключевых нововведений стали расширение пространственного диапазона измерения ионной температуры с помощью спектроскопии перезарядки (CXRS - charge exchange recombination spectroscopy) по сравнению c предыдущими экспериментами [11] и модернизация комплекса анализаторов атомов перезарядки NPA (NPA neutral particle analyzer) [12]. В результате этого на установке появилась современная диагностическая база для анализа поведения ионного компонента плазмы. Настоящая работа посвящена первым результатам исследования ионного теплопереноса в разрядах с допол- нительным нагревом плазмы с помощью нейтральной инжекции на сферическом токамаке Глобус-М2 (УНУ „Сферический токамак Глобус-М“, входящей в состав ФЦКП „Материаловедение и диагностика в передовых технологиях“, уникальный идентификатор проекта RFMEFI62119X0021).

Эксперименты проводились в дейтериевой плазме при токе $200 \mathrm{kA}$, тороидальном магнитном поле $0.7 \mathrm{~T}$, среднехордовая плотность электронов на квазистационарной стадии разряда составляла $\sim 4 \cdot 10^{19} \mathrm{~m}^{-3}$. Вытянутость в данном разряде была достаточно низкая $k=1.55$. Для дополнительного нагрева плазмы использовался дейтериевый пучок атомов с энергией частиц $27 \mathrm{keV}$, мощность $0.75 \mathrm{MW}$.

Измерения ионной температуры $T_{i}$ с помощью CXRS осуществлялись по семи расположенным в экваториальной плоскости установки хордам наблюдения (рис. 1), пересекающим ось инжекции пучка в точках с координатами большого радиуса плазмы $R$, указанными в таблице. Подробности обработки сигналов CXRS приведены в [11]. Значения ионной температуры на границе плазмы $(R=55-59 \mathrm{~cm})$ рассчитываются на осно-

Хорды измерения ионной температуры для CXRS

\begin{tabular}{c|c}
\hline Номер хорды & $R, \mathrm{~cm}$ \\
\hline 1 & 36.3 \\
2 & 39.3 \\
3 & 42.4 \\
4 & 45.4 \\
5 & 48.2 \\
6 & 50.8 \\
7 & 53.3
\end{tabular}




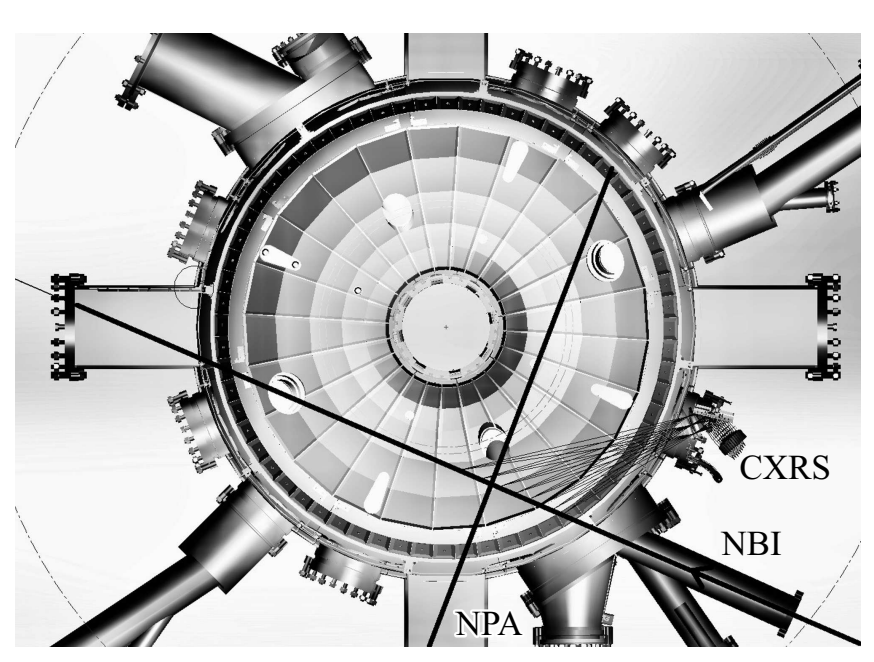

Рис. 1. Схема проведения эксперимента. Показаны линии наблюдения CXRS, направление наблюдения анализатора атомов перезарядки (NPA) и ось инжекции нейтрального пучка (NBI).

ве анализа пассивного сигнала CXRS, обусловленного излучением ионов $\mathrm{C}^{5+}$ из периферийной области плазменного шнура вблизи сепаратрисы. Зона локализации ионов $\mathrm{C}^{5+}$ является достаточно узкой, а максимум их концентрации расположен в области электронных температур порядка 100-130 eV. Измерения с помощью NPA АКОРД-24М проводились в течение всего разряда на $R=44.6 \mathrm{~cm}[12]$. Измерения профилей электронной температуры и плотности осуществлялись с помощью диагностики томсоновского рассеяния [13].

На рис. 2, $a$ представлен временной ход ионной температуры в центральной области плазмы в одном из разрядов экспериментальной серии \#38850, измеренной с помощью CXRS (хорда $R=45.4 \mathrm{~cm}$ ) и NPA (хорда $R=44.6 \mathrm{~cm})$. Видно, что результаты, полученные с помощью двух независимых диагностик, очень близки и лежат в пределах экспериментальной погрешности измерений. Также на рис. 2, $a$ представлены временной ход центральной электронной и ионной температуры, а также динамика теплового энергозапаса плазмы и среднехордовой плотности. Видно, что центральная ионная и электронная температура сравниваются через $15 \mathrm{~ms}$ после начала инжекции, а также, что электронная температура уменьшается вследствие значительного роста плотности (рис. 2). Энергозапас плазмы увеличивается с началом инжекции более чем в 2 раза. Однако величина теплового энергозапаса в целом невелика вследствие низкой вытянутости плазмы. Профили ионной температуры для моментов времени $t=175$ и $195 \mathrm{~ms}$ представлены на рис. $2, b$, на котором также приведен профиль электронной температуры, соответствующий квазистационарной стадии разряда $(t=196 \mathrm{~ms})$. В результате инжекции пучка ионная температура в центре увеличивается до $700 \mathrm{eV}$, и у профиля появляется выраженный максимум. Профиль электронной температуры в этом режиме на квазистационарной стадии разряда почти точно соответствует профилю ионной, в центре значение электронной температуры составляет около $700 \mathrm{eV}$.
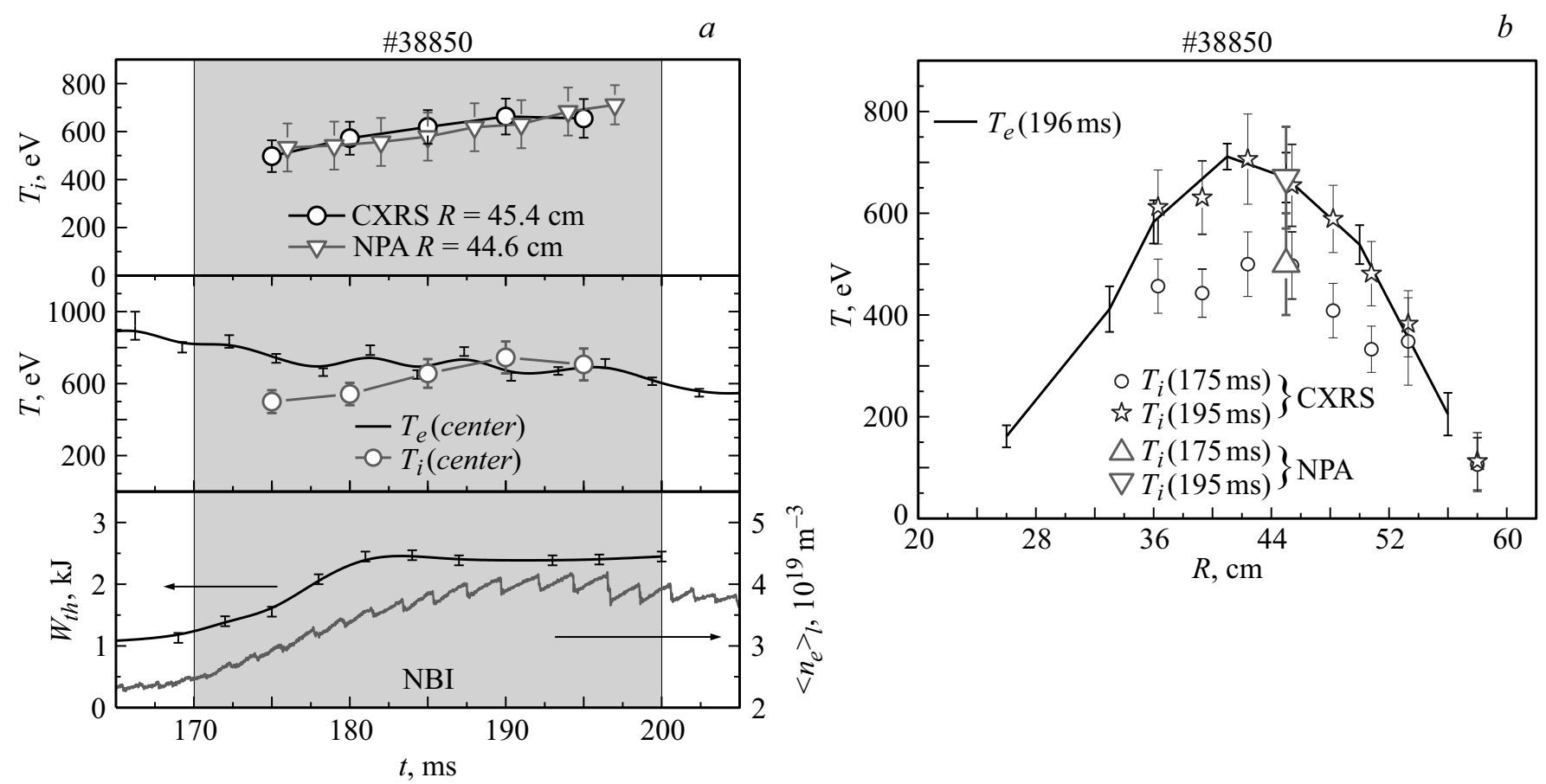

Рис. 2. $a-$ временной ход параметров плазмы: ионной температуры, измеренной с помощью CXRS (хорда $R=45.4 \mathrm{~cm})$ и NPA (хорда $R=44.6 \mathrm{~cm}$ ); центральной ионной и электронной температуры; теплового энергозапаса плазмы и среднехордовой плотности. $b-$ изменение во времени профиля ионной температуры и профиль электронной температуры. 

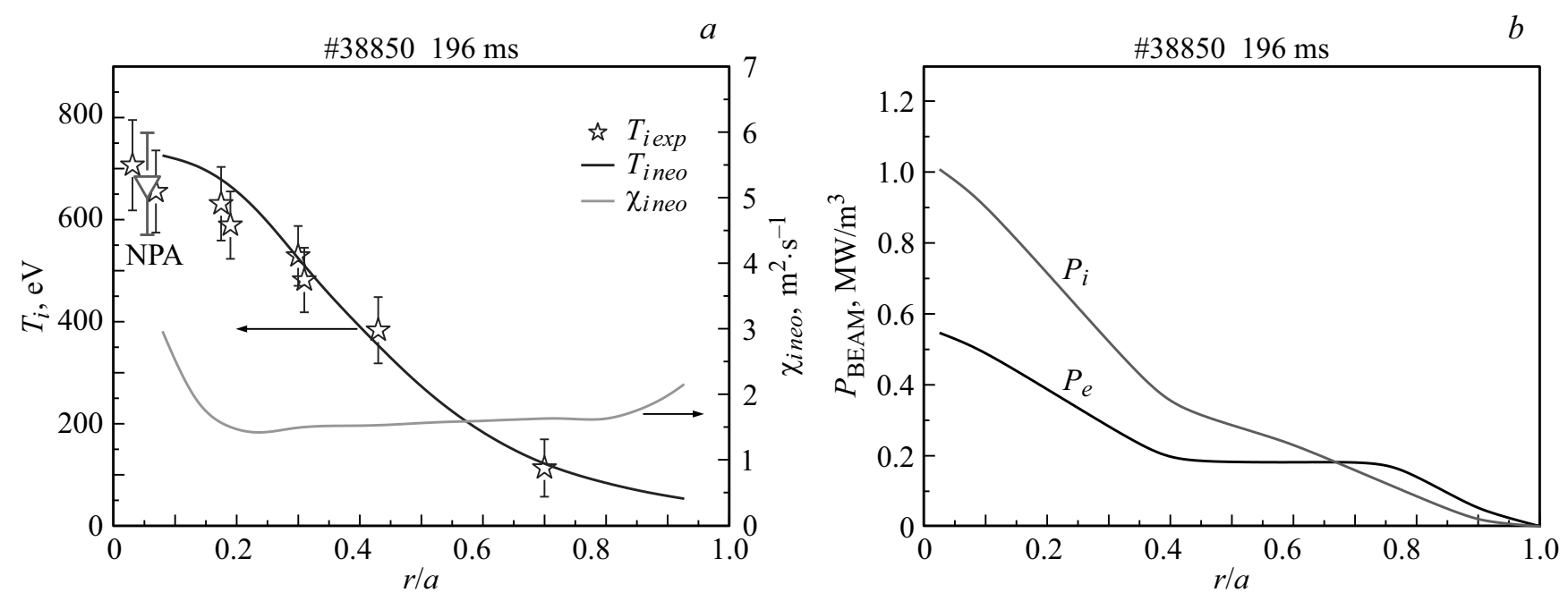

Рис. 3. $a-$ профили ионной температуры (экспериментальный и рассчитанный по неоклассической теории) и ионная температуропроводность 196 ms-разряда. $b$ - рассчитанные профили поглощенной мощности пучка для электронов и ионов.

Моделирование переноса тепла на основе полученных экспериментальных данных было проведено с помощью кода ASTRA [14]. При моделировании для ионного компонента плазмы решалась прямая транспортная задача: коэффициенты температуропроводности выбирались исходя из предположения о неоклассическом механизме переноса тепла ионами в плазме и рассчитывались с помощью блока NCLASS [15]. На основании этих расчетов строился профиль ионной температуры, который сравнивался с данными экспериментальных измерений (рис. $3, a)$. Для электронного компонента плазмы решалась обратная транспортная задача: на основании измеренных температуры и плотности плазмы рассчитывались коэффициенты температуропроводности. В качестве граничного условия для решения уравнения равновесия задавались параметры последней замкнутой магнитной поверхности, полученные с помощью кода EFIT [16]. Эффективный заряд плазмы был определен на основании измерения тормозного излучения плазмы и составлял $\sim 3$ (для $t=196 \mathrm{~ms}$ ) [17], основной примесью считался углерод. Поглощенная мощность пучка рассчитывалась с помощью блока NBI [18], при этом делались поправки на потери быстрых частиц [19]. Поглощенная мощность пучка составила порядка $210 \mathrm{~kW}$ $(120 \mathrm{~kW}$ поглотилось ионами, $90 \mathrm{~kW}$ - электронами), соответствующие профили приведены на рис. $3, b$. Полученные профили поглощенной мощности пучка схожи с профилями, получаемыми в расчетах для токамака Глобус-М, вследствие близких экспериментальных условий. Омическая мощность в разряде составляла около $260 \mathrm{~kW}$. Рассчитанный профиль ионной температуры $T_{i}$ (рис. $3, a$ ) хорошо согласуется с измеренным экспериментально, что подтверждает применимость неоклассической теории к описанию переноса тепла ионами в данном эксперименте. На рис. 3, $a$ также представлен соответствующий профилю $T_{i}$ коэффициент температуропроводности $\chi_{i \text { пео }}$.

Таким образом, на токамаке Глобус-М2 появился новый диагностический инструмент для анализа поведения ионного компонента плазмы (NPA и CXRS). Анализ накопленных данных показывает, что измерения ионной температуры с помощью CXRS хорошо согласуются с измерениями NPA. Результаты моделирования подтверждают выводы исследований, проводимых ранее на токамаке Глобус-М [4-7]. В дальнейшем на токамаке Глобус-М2 планируются эксперименты с параметрами, максимально близкими к проектным [8], что позволит более подробно исследовать ионный теплоперенос и проверить результаты предсказательного моделирования, приведенные в [8]. Проведенные в настоящей работе оценки транспортных процессов в сферическом токамаке Глобус-М2 в текущих экспериментальных условиях демонстрируют преимущественно неоклассический ионный теплоперенос.

\section{Финансирование работы}

Работа выполнена в рамках государственного задания Министерства науки и высшего образования РФ (№ 0040-2019-0023).

\section{Конфликт интересов}

Авторы заявляют, что у них нет конфликта интересов.

\section{Список литературы}

[1] H. Meyer, A.R. Field, R.J. Akers, C. Brickley, N.J. Conway, A. Patel, P.G. Carolan, C. Challis, G.F. Counsell, G. Cunningham, P. Helander, A. Kirk, B. Lloyd, R. Maingi, M.R. Tournianski, M.J. Walsh and the MAST and NBI teams, Plasma. Phys. Control. Fusion, 46, A291 (2003). doi.org/10.1088/0741-3335/46/5A/032 
[2] A.R. Field, R.J. Akers, D.J. Applegate, C. Brickley, P.G. Carolan, C. Challis, N.J. Conway, S.C. Cowley, G. Cunningham, N. Joiner, H. Meyer, A. Patel, C. Roach, M. Valovič, M.J. Walsh and the MAST team, in 20th IAEA Fusion Energy Conf. (Vilamoura, Portugal, 2004), EX/P2-11. https://citeseerx.ist.psu.edu/viewdoc/download?doi $=10.1 .1 .483 .743 \& \mathrm{rep}=$ rep $1 \&$ type $=$ pdf

[3] S.M. Kaye, F.M. Levinton, D. Stutman, K. Tritz, H. Yuh, M.G. Bell, R.E. Bell, C.W. Domier, D. Gates, W. Horton, J. Kim, B.P. LeBlanc, N.C. Luhmann, Jr, R. Maingi, E. Mazzucato, J.E. Menard, D. Mikkelsen, D. Mueller, H. Park, G. Rewoldt, S.A. Sabbagh, D.R. Smith, W. Wang, Nucl. Fusion, 47, 499 (2007). doi.org/10.1088/0029$5515 / 47 / 7 / 001$

[4] G.F. Avdeeva, I.V. Miroshnikov, N.N. Bakharev, G.S. Kurskiev, M.I. Patrov, V.Yu. Sergeev, P.B. Schegolev, J. Phys.: Conf. Ser., 666, 012002 (2016). doi.org/10.1088/17426596/666/1/012002

[5] А.Ю. Тельнова, Г.С. Курскиев, И.В. Мирошников, Г.Ф. Авдеева, Н.Н. Бахарев, В.К. Гусев, В.Б. Минаев, А.Д. Мельник, Ю.В. Петров, Н.В. Сахаров, Ф.В. Чернышев, П.Б. Щёголев, Письма в ЖТФ, 44 (15), 100 (2018). DOI: 10.21883/PJTF.2018.15.46447.17330 [Пер. версия: 10.1134/S1063785018080126].

[6] A.Yu. Telnova, G.S. Kurskiev, I.V. Miroshnikov, N.V. Sakharov, E.O. Kiselev, M.M. Larionova, N.N. Bakharev, D.M. Larionova, V.K. Gusev, N.A. Khromov, V.B. Minaev, M.I. Patrov, Yu.V. Petrov, A.D. Sladkomedova, P.B. Shchegolev, V.A. Tokarev, S.Yu. Tolstyakov, E.A. Tukhmeneva, Plasma Phys. Control. Fusion, 62, 045011 (2020). doi.org/10.1088/1361-6587/ab6da5

[7] I.Yu. Senichenkov, V.A. Rozhansky, A.V. Bogomolov, V.K. Gusev, N.V. Sakharov, Yu.V. Petrov, V.B. Minaev, S.Yu. Tolstyakov, M.I. Patrov, F.V. Chernyshev, B.B. Ayushin, G.S. Kurskiev and the Globus-M team, in Proc. 35th EPS Conf. on plasma physics. Europhysics conference abstracts (Hersonissos, 2008), vol. 32D, P-2.046 http://epsppd.epfl.ch/Hersonissos/pdf/P2_046.pdf

[8] V.B. Minaev, V.K. Gusev, N.V. Sakharov, V.I. Varfolomeev, N.N. Bakharev, V.A. Belyakov, E.N. Bondarchuk, P.N. Brunkov, F.V. Chernyshev, V.I.Davydenko, V.V. Dyachenko, A.A. Kavin, S.A. Khitrov, N.A. Khromov, E.O. Kiselev, A.N. Konovalov, V.A. Kornev, G.S. Kurskiev, A.N. Labusov, A.D. Melnik, A.B. Mineev, M.I. Mironov, I.V. Miroshnikov, M.I. Patrov, Yu.V. Petrov, V.A. Rozhansky, A.N. Saveliev, I.Yu. Senichenkov, P.B. Shchegolev, O.N. Shcherbinin, I.V. Shikhovtsev, A.D. Sladkomedova, V.V. Solokha, V.N. Tanchuk, A.Yu. Telnova, V.A. Tokarev, S.Yu. Tolstyakov, E.G. Zhilin, Nucl. Fusion, 57, 066047 (2017). doi.org/10.1088/1741-4326/aa69e0

[9] В.К. Гусев, В.Е. Голант, Е.3. Гусаков, В.В. Дьяченко, М.А. Ирзак, В.Б. Минаев, Е.Е. Мухин, А.Н. Новохацкий, К.А. Подушникова, Г.Т. Раздобарин, Н.В. Сахаров, Е.Н. Трегубова, В.С. Узлов, О.Н. Щербинин, В.А. Беляков, А.А. Кавин, Ю.А. Косцов, Е.Г. Кузьмин, В.Ф. Сойкин, Е.А. Кузнецов, В.А. Ягнов, ЖТФ, 69 (9), 58 (1999). https://journals.ioffe.ru/articles/viewPDF/36172 [Пер. версия: 10.1134/1.1259469].

[10] Н.Н. Бахарев, И.М. Балаченков, В.И. Варфоломеев, А.В. Воронин, В.К. Гусев, В.В. Дьяченко, М.В. Ильясова, Е.О. Киселев, А.Н. Коновалов, Г.С. Курскиев, А.Д. Мельник, В.Б. Минаев, И.В. Мирошников, А.Н. Новохацкий, М.И. Патров, Ю.В. Петров, Н.В. Сахаров, О.М. Скрекель,
А.Ю. Тельнова, В.А. Токарев, С.Ю. Толстяков, Е.А. Тюхменева, Е.М. Хилькевич, Н.А. Хромов, Ф.В. Чернышев, И.Н. Чугунов, А.Е. Шевелев, П.Б. Щеголев, Физика плазмы, 46 (7), 579 (2020). DOI: 10.31857/S036729212007001X [Пер. версия: 10.1134/S1063780X20070016].

[11] M.M. Larionova, I.V. Miroshnikov, V.K. Gusev, V.B. Minaev, M.I. Patrov, Yu.V. Petrov, N.V. Sakharov, P.B. Schegolev, A.Yu. Telnova, N.N. Bakharev, J. Phys.: Conf. Ser., 1400, 077018 (2019). https://doi.org/10.1088/1742-6596/1400/7/077018

[12] N.N. Bakharev, F.V. Chernyshev, V.K. Gusev, E.O. Kiselev, G.S. Kurskiev, M.M. Larionova, A.D. Melnik, V.B. Minaev, M.I. Mironov, I.V. Miroshnikov, Yu.V. Petrov, N.V. Sakharov, P.B. Shchegolev, O.M. Skrekel, A.Yu. Telnova, E.A. Tukhmeneva, V.I. Varfolomeev, Plasma Phys. Control. Fusion, 62, 125010 (2020). https://doi.org/10.1088/13616587/abbe32

[13] Г.С. Курскиев, С.Ю. Толстяков, А.А. Березуцкий, В.К. Гусев, М.М. Кочергин, В.Б. Минаев, Е.Е. Мухин, М.И. Патров, Ю.В. Петров, Н.В. Сахаров, В.В. Семёнов, П.В. Чернаков, ВАНТ. Сер.: Термоядерный синтез, вып. 2, 81 (2012). http://vant.iterru.ru/vant_2012_2/7.pdf

[14] G.V. Pereverzev, P.N. Yushmanov, ASTRA (Automated System for TRansport Analysis), Max-Plank report IPP 5/98 (2002). https://w3.pppl.gov/ hammett/work/2009/Astra_ocr.pdf

[15] W.A. Houlberg, K.C. Shaing, S.P. Hirshman, M.C. Zarnstorff, Phys. Plasmas, 4, 3230 (1997). doi.org/10.1063/1.872465

[16] L.L. Lao, H.St. John, R.D. Stambaugh, A.G. Kellman, W. Pfeiffer, Nucl. Fusion, 25, 1611 (1985). doi.org/10.1088/0029-5515/25/11/007

[17] E.A. Tukhmeneva, S.Yu. Tolstyakov, G.S. Kurskiev, V.K. Gusev, V.B. Minaev, Yu.V. Petrov, N.V. Sakharov, A.Yu. Telnova, N.N. Bakharev, P.B. Shchegolev, E.O. Kiselev, Plasma Sci. Technol., 21, 105104 (2019). doi.org/10.1088/2058-6272/ab305f

[18] A. Polevoi, H. Shirai, T. Takizuka, JAERI -Data/Code 97-014 (1997).

[19] N.N. Bakharev, F.V. Chernyshev, P.R. Goncharov, V.K. Gusev, A.D. Iblyaminova, V.A. Kornev, G.S. Kurskiev, A.D. Melnik, V.B. Minaev, M.I. Mironov, M.I. Patrov, Yu.V. Petrov, N.V. Sakharov, P.B. Shchegolev, S.Yu. Tolstyakov, G.V. Zadvitskiy, Nuclear Fusion, 55, 043023 (2015). doi.org/10.1088/0029-5515/55/4/043023 